\title{
Eficiencia de las universidades estatales colombianas
}

\author{
Efficiency of the colombian state universities \\ villar32@hotmail.com \\ Josefa Ramoni Perazzib \\ j.ramoni@udes.edu.co
}

Andrea González Villar

Giampaolo Orlandoni Merlic

gorlandoni@udes.edu.co

\begin{abstract}
Resumen
Esta investigación presenta el análisis de la evolución de los niveles de eficiencia técnica de las universidades estatales colombianas. Para ello se toma la información del Sistema de Indicadores de Gestión propuesto por el SUE y el MEN, evaluado en las 32 universidades públicas durante el período 2003-2012. Previo a la estimación de la eficiencia, se obtienen los índices de resultados de capacidad, formación e investigación, a través de los puntajes factoriales del análisis factorial. La estimación de la eficiencia técnica en los índices de resultados de formación e investigación en función del índice de resultados de capacidad, se hace bajo un modelo de fronteras estocásticas de producción con datos panel. El modelo asume los efectos de ineficiencia técnica variantes en el tiempo. La clasificación de las universidades se establece con las predicciones lineales de la regresión cuantíl, en los cuartiles 25, 50 y 75 , que definen las universidades con eficiencia alta, media y baja, respectivamente. Los resultados de esta investigación indican que los índices de formación e investigación están determinados positivamente por los recursos financieros, la planta física y la planta profesoral, esta última con mayor influencia.
\end{abstract}

Palabras clave: Indicadores de gestión, modelo de fronteras estocásticas, datos panel.

\footnotetext{
Abstract

This study analyses the evolution of the technical efficiency of the public universities in Colombia. The paper uses the information provided by the System of

${ }^{a}$ Licenciada en Estadística - IEAC-Universidad de Los Andes, Mérida- Venezuela y Universidad Nacional Experimental del Sur del Lago, Santa Bárbara del Zulia, Venezuela.

${ }^{b}$ Profesora - Universidad de Santander, Colombia.

${ }^{\mathrm{c}}$ Profesor - Universidad de Santander, Colombia
} 


\section{Andrea González Villar, Josefa Ramoni Perazzi \& Giampaolo Orlandoni Merli}

Management Indicators developed by the government, related to 32 public universities during the period 2003-2012. The factorial scores of factor analysis are used to estimate the efficiency, education and research indexes used in the study. To estimation the education and research technical efficiency, a panel data stochastic production frontier model is used, controlling for the capacity index. The model assumes time-varying technical inefficiency. Finally, quantile regression is used to classify universities on quartiles 25, 50 and 75, based on their high, medium or low efficiency level. The results indicate that both education and research indexes increase with financial resources, physical capacity and, especially, professorial capacity.

Keywords: Management indicators, stochastic frontier model, data panel.

\section{Introducción}

Las instituciones de educación superior colombianas con financiamiento público, reciben recursos económicos del Estado de acuerdo con su desempeño en relación a su misión, su carácter de universidad pública, así como sus recursos humanos y de infraestructura disponibles. Estas instituciones tienen una asignación básica presupuestaria anual reglamentada en el Artículo 87 de la Ley 30 de 1992. Dicha asignación de recursos económicos se reajusta de acuerdo con la situación económica del país, reflejada en el alza del costo de la vida. Es por esto que, el Sistema de Universidades Estatales (SUE) y el Ministerio de Educación Nacional (MEN) han creado el sistema de indicadores de gestión, para evaluar y clasificar a las universidades de acuerdo con los resultados obtenidos en las actividades misionales. Por tal razón, el SUE ha orientado sus esfuerzos en el análisis de la eficiencia de las universidades estatales colombianas en los últimos años. En este sentido, el sistema de indicadores de gestión está conformado por el índice de capacidad, el cual mide los recursos humanos, financieros y físicos; el índice de formación, que mide la mutidisciplinariedad, productividad, cobertura, impacto y calidad de los estudiantes egresados de estas universidades; el índice de investigación, que mide la productividad y el impacto de la investigación; el índice de extensión, que mide la actividad de extensión y el índice de bienestar estudiantil. La distribución del presupuesto se hace de acuerdo con las ponderaciones de cada uno de los componentes o índices, los cuales están formados por una serie de indicadores que definen y muestran características de cada universidad, siendo los índices de formación e investigación los de mayor peso en esta ponderación. Aparte de esta distribución presupuestal, aquellas universidades acreditadas reciben un aporte adicional a su presupuestq ${ }^{1}$

El propósito de esta investigación es analizar la evolución en el tiempo de la eficiencia técnica de las universidades estatales colombianas. Se entiende por eficiencia

\footnotetext{
${ }^{1}$ Ver los documentos Metodología para la Distribución de Recursos del Artículo 87 de la Ley 30 de 1992 del Viceministerio de Educación Superior, Ministerio de Educación Nacional. República de Colombia y Propuesta Metodológica para la Distribución de Recursos del Artículo 87 de la Ley 30 de 1992 del Ministerio de Educación Nacional. República de Colombia
} 
técnica, la capacidad que tiene un proceso para generar la mayor cantidad de producto posible (en este caso, profesionales e investigación), dado los recursos de que dispone. Para el logro de este objetivo, el estudio aplica modelos de fronteras estocásticas al conjunto de indicadores de para cada universidad durante el período 2003-2012.

A través de esta metodología se busca identificar cuáles son las universidades más eficientes con respecto a los índices de formación e investigación del sistema de indicadores de gestión, señalando cómo la capacidad, definida en términos de recursos disponibles, influye directamente sobre el rendimiento de la universidad en todos sus ámbitos. Los resultados apuntan a que las universidades del Tolima, Militar de Nueva Granada y Valle son las más eficientes en cuanto a formación y Universidad Nacional de Colombia lo es en investigación.

\section{Aspectos preliminares}

En economía, se entiende por "eficiencia técnica" la capacidad para hacer un aprovechamiento exhaustivo de los recursos disponibles, a fin de obtener de ellos la máxima producción posible. Para el caso que nos ocupa, esa producción vendrá medida en términos de recursos formados e investigación generada. Las herramientas fundamentales para el análisis de la eficiencia técnica son el Análisis Envolvente de Datos (DEA, por sus siglas en inglés) y los modelos de fronteras estocásticas. El primero consiste en un método no paramétrico, que recurre a la programación lineal para estudiar la relación entre insumos y productos, asumiendo libre disponibilidad de estos, así como convexidad y rendimientos constantes y variables a escala. Los modelos de fronteras estocásticas, los cuales se explican a continuación, constituyen la alternativa paramétrica, para la cual se requiere especificar relaciones funcionales específicas, siendo la más común la función de producción Cobb-Douglas.

Varios han sido los intentos por analizar la eficiencia de las universidades públicas a nivel mundial. Ramos et al. (2015) llevan a cabo un resumen exhaustivo de los diferentes estudios sobre este particular, agrupados según la metodología aplicada, de lo que se concluye que el DEA ha sido el método más comúnmente utilizado, seguido de los modelos de fronteras estocásticas, probablemente porque el primero es relativamente menos demandante en términos de cantidad de información. Específicamente, Cervera et al. (2013) resumen las aplicaciones de DEA para el caso de nuestro país. Melo et al. (2014) aplican fronteras estocásticas para cuantificar el impacto positivo que el capital físico y humano tienen sobre el logro académico medido a través de los resultados de las pruebas Saber Pro. El propio estudio de Ramos y colaboradores recurren a DEA para analizar la eficiencia técnica de las universidades públicas colombianas en diferentes periodos de tiempo. Adicionalmente el MEN (2013), propone un modelo de Indicadores de Gestión para las universidades públicas en Colombia a partir de las fronteras estocásticas de producción para el año 2011. No obstante, no existen para el momento en el 


\section{Andrea González Villar, Josefa Ramoni Perazzi \& Giampaolo Orlandoni Merli}

país estudios que apliquen modelos de datos panel de fronteras estocásticas para analizar la eficiencia de las universidades públicas.

\section{Metodología}

Esta investigación es de tipo cuantitativa de corte longitudinal, en la que se analiza el sistema de indicadores de gestión propuesto por el SUE-MEN, medido a las universidades estatales de Colombia durante el período 2003-2012. Los índices analizados son: índice de capacidad, índice de formación y el índice de investigación. La base de datos original es de tipo panel, de la cual se descartan todos aquellos indicadores que no hayan sido medidos durante este período, en virtud de no afectar los resultados de la evolución de las universidades en el tiempo. Resultando finalmente una estructura de datos de panel balanceado, donde cada variable ha sido medida en todas las universidades en el período considerado. Los datos de cada uno de los índices son procesados y analizados usando el software estadístico Stata IC 11.0.

Se parte de la construcción de los índices de resultados de formación, capacidad e investigación, los cuales se generan a través de los coeficientes de los puntajes factoriales del análisis factorial. Estos coeficientes forman una combinación lineal con cada indicador que hace parte del índice en cuestión, evaluando el mismo sobre cada uno de los individuos, y produciendo una nueva variable (Rencher 2002).

En la estimación y análisis de la eficiencia de las universidades se utiliza la metodología de modelos de fronteras estocásticas de producción con datos panel, con variables de tipo cuantitativas, para estudiar los factores de capacidad que afectan la eficiencia técnica de las universidades tanto en el índice de resultado de formación como en el índice de resultado de investigación. En este caso la eficiencia técnica se asume variante en el tiempo.

\subsection{Análisis de fronteras estocásticas de producción estocásti- ca con datos panel}

El análisis de fronteras estocásticas (AFE) se inició en la segunda mitad de la década de los 70, como técnica econométrica para la medición de la eficiencia y la productividad directamente influenciada por las versiones paramétricas de medición de la eficiencia económica, inspiradas en el trabajo pionero de Farrell en el año 1957 (Castilla \& García 2009). Esta investigación se orienta al análisis de la eficiencia técnica, definida como el logro del máximo producto o servicio con un costo dado, originado por una combinación específica de factores (Gómez 2006).

La estimación sobre datos de tipo panel es favorable para el análisis de la eficiencia de una empresa y para el desarrollo de nuevas técnicas de estimación. En términos generales, los datos tipo panel contienen información para cada empresa en varios periodos de tiempo, lo cual no ocurre con datos de corte transversal, donde se 
tiene información de una empresa en un punto específico del tiempo (Montoya et al. 2002).

Los modelos considerados en esta investigación son los propuestos por Battese \& Coelli (1992) y Battese \& Coelli (1995). Estos modelos suponen en forma general que los datos consisten en una muestra de $N$ firmas en $T$ períodos.

La frontera estocástica de producción (FEP) generalizada se define como:

$$
y_{i t}=\exp \left(x_{i t} \beta+v_{i t}-u_{i t}\right)
$$

donde $y_{i t}$ representa el producto de la $i$-ésima empresa en el período $t, x_{i t}$ es un vector $1 \times k$ cuyos valores son funciones de los inputs y de otras variables explicativas para la $i$-ésima empresa en el momento $t$. $\beta$ es un vector $k \times 1$ de parámetros a estimar. Los $v_{i t}$ son errores aleatorios independientes e idénticamente distribuidos (i.i.d.) como una normal con media cero y varianza constante $\sigma_{v}^{2}$. Los $u_{i t}$ son variables aleatorias no negativas y no observables asociadas con la ineficiencia técnica de la producción.

Battese \& Coelli (1992), definen los $u_{i t}$ como:

$$
u_{i t}=\eta_{i t} u_{i}=\exp \left(-\eta\left(t-T_{i}\right)\right) u_{i}
$$

donde $\eta$ es un parámetro desconocido a estimar, y los $u_{i}(i=1, \cdots, N)$ son variables aleatorias i.i.d. no negativas, que provienen de una distribución normal truncada (en cero) con media $\mu$ y varianza $\sigma_{u}^{2}$ (desconocidas). Este modelo supone que los efectos de ineficiencia técnica en los períodos iniciales, para los $N$ individuos del panel, son una función exponencial de los efectos de ineficiencia correspondientes al último período (notarse que $u_{i t}=u_{i}$ ). En este sentido, se plantea la hipótesis nula de que los efectos de ineficiencia técnica no están presentes en el modelo, es decir, $H_{0}: \gamma=0$.

El parámetro $\gamma$ se define como:

$$
\gamma=\frac{\sigma_{u_{i}}^{2}}{\sigma_{v}^{2}+\sigma_{u_{i}}^{2}}
$$

Asimismo, las hipótesis nulas de que los efectos de ineficiencia son invariantes en el tiempo y que tienen una distribución seminormal son definidas por $H_{0}: \eta=0$ y $H_{0}: \mu=0$, respectivamente. Estas hipótesis se contrastan mediante un test de razón de verosimilitud generalizado (Likelihood-ratio test o LR), cuyo estadístico de prueba se define como:

$$
\gamma=-2 \ln \left[\frac{L\left(H_{0}\right)}{L\left(H_{1}\right)}\right]
$$

donde $H_{0}$ y $H_{1}$ son las hipótesis nula y alternativa respectivamente. Si la $H_{0}$ es verdadera, entonces $\gamma$ se distribuye asintóticamente como una Ji cuadrado. Si la $H_{0}$ incluye a $\gamma=0$, entonces $\gamma$ se distribuye como una Ji cuadrado combinada (Coelli 1996). 


\section{Andrea González Villar, Josefa Ramoni Perazzi \& Giampaolo Orlandoni Merli}

Battese \& Coelli (1995) define los $u_{i t}$, como:

$$
u_{i t}=z_{i t} \delta+W_{i t}
$$

donde, $z_{i t}$ es un vector $1 \times m$ de variables explicativas asociadas a los efectos de ineficiencia técnica, $\delta$ es un vector $m \times 1$ de parámetros a estimar, y las $W_{i t}$ son un conjunto de variables aleatorias no observables que se suponen (i.i.d.) provenientes de una distribución normal truncada con media cero y varianza $\sigma^{2}$, de forma tal que los $u_{i t}$ son no negativos, $W_{i t} \geq-z_{i t} \delta$.

La hipótesis nula de que los efectos de ineficiencia son no aleatorios se expresa como: $H_{0}: \delta=0$. El parámetro $\delta$ se define tal como se muestra en $(3)$. La hipótesis nula de que los efectos de ineficiencia no están afectados por las variables explicativas $z_{i t}$ se plantea como $H_{0}: \delta^{\prime}=0$, donde $\delta^{\prime}$ representa el vector de parámetros $\delta$ excluido el intercepto.

El modelo con eficiencia técnica variante en el tiempo se puede plantear bajo el supuesto de que $f\left(x_{i t} ; \delta\right)$ toma la forma loglineal del tipo Cobb-Douglas, y se puede escribir de la siguiente forma:

$$
\ln y_{i t}=\beta_{0 t}+\sum \beta_{n} \ln x_{n_{i t}}+v_{i t}-u_{i t}
$$

donde, $y_{i t}$ denota el producto de la $i$-ésima unidad en el período $t, x_{n_{i t}}$ representa un vector con los valores correspondientes a las inputs y otras variables relevantes, y $\beta$ corresponde a un vector de parámetros por estimar. $\beta_{0 t}$ es el intercepto de la frontera de producción común a todas las empresas en el periodo $t$. El término $n_{i t}$ corresponde al componente de ruido bajo el supuesto de tener una distribución i.i.d del tipo $N\left(0, \sigma_{v}^{2}\right)$ a lo largo de todos los individuos y del tiempo, además de ser independiente tanto de las variables explicativas $x_{n_{i t}}$ como también de los errores $u_{i t}$, los cuales son el componente no negativo de ineficiencia técnica variante en el tiempo, del término de error.

La eficiencia técnica media puede calcularse partiendo del supuesto de que los $u_{i t}$ son i.i.d. $N\left(0, \sigma^{2}\right)$, truncada en cero, entonces la eficiencia media es:

$$
E\left[\exp \left(-u_{i t}\right)\right]=2\left[1-\Phi\left(\sigma_{s} \sqrt{\gamma}\right)\right] \exp \left(-\gamma \sigma_{s}^{2} / 2\right)
$$

La eficiencia técnica para cada firma se define como: $E T_{i}=\exp \left(-u_{i t}\right)$, sin embargo debe notarse que los efectos de la ineficiencia técnica, $u_{i t}$ son inobservables. Conocidos los parámetros $\beta$ de la frontera estocástica de producción, sólo la diferencia, $e_{i t}=v_{i t}-u_{i t}$ puede ser observada. El mejor predictor para $u_{i t}$, es la esperanza condicional de $u_{i t}$, dado el valor de $v_{i t}-u_{i t}$.

Battese \& Corra (1977) señalan que el mejor predictor de $\exp \left(-u_{i t}\right)$ puede obtenerse utilizando:

$$
E\left[\exp \left(u_{i t}\right)\right]=\frac{1-\Phi\left(\sigma_{A}+\gamma e_{i} / \sigma_{A}\right)}{1-\Phi\left(\gamma e_{i} / \sigma_{A}\right)} \exp \left(\gamma e_{i}+\sigma_{A}^{2} / 2\right)
$$

Comunicaciones en Estadística, junio 2017, Vol. 10, No. 2 
donde $\sigma_{A}=\sqrt{\gamma(1-\gamma) \sigma_{s}^{2}}, e_{i}=\ln y_{i}-x_{i} \beta, \Phi(\cdot)$ es la función de densidad de una variable aleatoria con una distribución normal estandarizada.

Luego de estimar la eficiencia en formación e investigación de las universidades en los años 2003 a 2012, se utiliza la regresión cuantíl, con el fin de obtener los valores predichos de la regresión lineal en los cuartiles 25, 50 y 75. Estas predicciones son graficadas en conjunto con la eficiencia de cada indicador respecto a la capacidad de la universidad. De acuerdo a la posición que ocupe cada universidad en este gráfico, la misma será categorizada como eficiencia alta, media y baja.

\section{Resultados}

El análisis de la evolución de la eficiencia de las universidades estatales colombianas en sus procesos de formación e investigación durante el período 2003-2012, requiere de una serie de tratamientos estadísticos previos a la estimación de la eficiencia técnica. En virtud de la importancia individual que tienen estos índices, este análisis se hace de manera independiente para cada uno de ellos.

\subsection{Análisis del índice de formación}

La estimación de la eficiencia que muestran las universidades en el período 2003 a 2012, sobre el IRF ${ }^{2}$ se obtiene del modelo de fronteras estocásticas de producción con datos panel, en el que se expresa el índice de resultados de formación como una función de los indicadores del índice de capacidad.

El modelo teórico viene dado por:

$$
L I R F_{i t}=\beta_{0}+\beta_{1} \text { Lecur }_{i t}+\beta_{2} \text { LDocent }_{i t}+\beta_{3} \text { LMetros }_{i t}+\beta_{4} L_{\text {Admin }}{ }_{i t}+\left(v_{i t}-u_{i t}\right)
$$

donde el subíndice $i$ representa las universidades, $t$ los años, Recur representa la variable recursos, Docent representa la variable de docentes, Metros representa el área en metros y Admin representa la variable de administración.

Siendo el índice de resultados de formación una representación de la actividad docente en una institución de educación superior, es de esperarse que el número de docentes en las mismas afecte de manera directa al índice de resultados de formación. Además, se sabe que el tamaño de la planta física de la universidad, los gastos administrativos y recursos financieros como indicadores de capacidad, pudiera contribuir con la labor docente, por lo que no se deja de lado la posibilidad de que la misma afecte al IRF de manera directa.

Las estimaciones de máxima verosimilitud para los parámetros de la FEP de las universidades estatales colombianas, resultaron ser todas estadísticamente significativas (tabla 1). Igualmente, el parámetro de ineficiencia técnica es significativo,

\footnotetext{
${ }^{2}$ Algunos de los valores del IRF resultaron ser negativos, por lo que se llevó a cabo un cambio de origen, a fin de poder transformar la variable en términos logarítmicos.
}

Comunicaciones en Estadística, junio 2017, Vol. 10, No. 2 
90 Andrea González Villar, Josefa Ramoni Perazzi \& Giampaolo Orlandoni Merli

Tabla 1: Resumen de las estimaciones del Modelo Final para el IRF. Fuente: elaboración propia.

\begin{tabular}{llllll}
\hline Parámetro & $\begin{array}{l}\text { Coeficiente } \\
\text { Estimado }\end{array}$ & $\begin{array}{l}\text { Desviación } \\
\text { Estándar }\end{array}$ & $z$ & $\begin{array}{l}\text { valor } \\
p\end{array}$ & $\begin{array}{l}\text { Coeficiente } \\
\text { Estandar. }\end{array}$ \\
\hline$\beta_{1}$ & 0.104 & 0.049 & 2.100 & 0.035 & 0.014 \\
$\beta_{2}$ & 0.462 & 0.071 & 6.480 & 0.000 & 0.092 \\
$\beta_{3}$ & 0.163 & 0.050 & 3.210 & 0.001 & 0.232 \\
\hline$\mu$ & 5.717 & 0.710 & 8.050 & 0.000 & \\
$\eta$ & 0.005 & 0.001 & 3.300 & 0.001 & \\
llgt $Y$ & 1.273 & 0.361 & 3.530 & 0.000 & \\
$Y$ & 0.781 & 0.033 & & & \\
\hline
\end{tabular}

indicando que los efectos de ineficiencia técnica tienen un impacto en el IRF. Bajo la hipótesis de que estos efectos tienen una distribución seminormal el parámetro $\mu$ es significativo. El parámetro de efectos de ineficiencia técnica variante en el tiempo $(\eta)$, resultó significativo, revelando que el nivel de ineficiencia técnica de las universidades estatales colombianas cambia año a año y de forma decreciente.

El aumento en los recursos económicos afecta de manera directa al IRF, y al aumentar en $1 \%$ en promedio, el índice de resultados de formación se incrementa en un $0,104 \%$. El incremento en el número de docentes tiene un efecto directo sobre el IRF, debido a que es un factor importante en el desarrollo de la formación académica de las universidades. Al incrementarse en $1 \%$ la planta profesoral, el IRF crecerá en promedio en un $0,462 \%$. Y al aumentar $1 \%$ en promedio los metros cuadrados de la universidad, el IRF se incrementará 0,163\%; acotando que este indicador el que tiene un mayor efecto sobre el índice en cuestión. La figura 4.1 muestra el comportamiento de la eficiencia de este indicador en las universidades año a año, contra el índice de capacidad.

Obsérvese que en general las pendientes de la regresión cuantíl cambian al aumentar la capacidad. Así, las instituciones que tienen niveles de eficiencia por encima de cuartil 0.75 , forman el grupo de las universidades con eficiencia alta en el índice de formación. En este mismo orden de ideas, es factible construir un segundo grupo de instituciones ubicadas entre los cuartiles 0.25 y 0.75 , denominado "universidades de eficiencia media", y en último lugar se define el grupo de universidades de eficiencia baja constituido por aquellas que se localizan por debajo del cuartil 0.25.

Es importante resaltar que las universidades Nacional de Colombia (UNAL) y Antioquia (UDEA), se alejan considerablemente del resto de las instituciones, debido a que son las de mayor tamaño, en términos de su capacidad. Las predicciones lineales de la regresión cuantíl clasifican estas universidades como de eficiencia media, con niveles estables a lo largo del tiempo. Acotando que, para el año 2003 la UDEA es más eficiente que la UNAL, pues la pendiente de la eficiencia técnica de la UDEA está ligeramente por encima de la UNAL, situación que se atenúa con el paso de los años. 


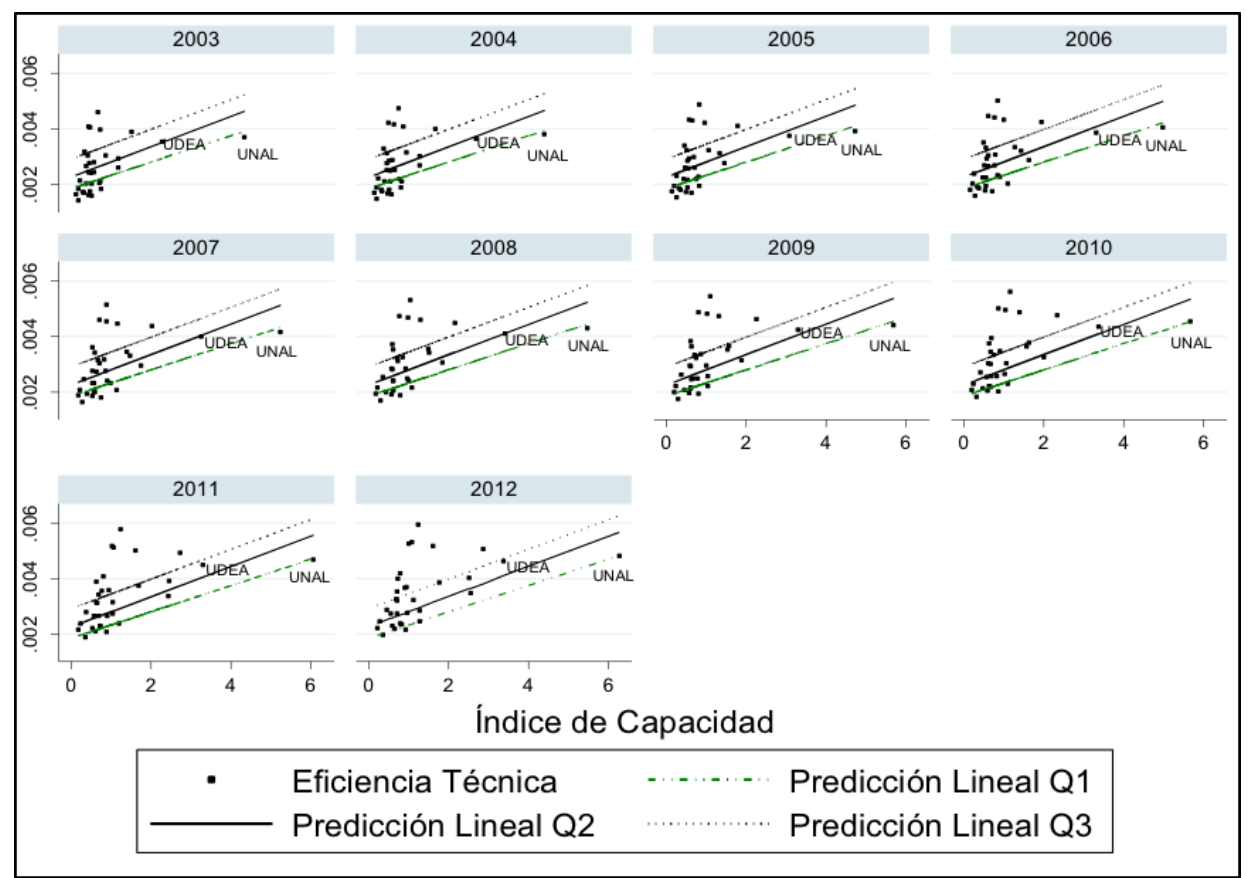

Figura 1: Disposición de la frontera estocástica de producción del IRF a través de la regresión cuantíl, para las universidades estatales colombianas durante el período 2003-2012. Datos MEN. Fuente: elaboración propia.

\subsection{Análisis del Índice de Investigación}

La estimación de la eficiencia que muestran las universidades durante el período 2003 a 2012, sobre el índice de resultados de investigación (IRI) se obtiene del modelo de fronteras estocásticas de producción con datos panel, en el que se expresa el IRI en función del índice de capacidad.

El modelo teórico viene dado por:

$$
L_{I R I_{i t}}=\beta_{0}+\beta_{1} \text { LRecfin }_{i t}+\beta_{2} \text { LDocent }_{i t}+\beta_{3} \text { LMetros }_{i t}+\beta_{4} L A d \min _{i t}+\left(v_{i t}-u_{i t}\right)
$$

donde el subíndice $i$ representa las universidades, $t$ los años, RecFin representa la variable recursos financieros, Docent representa la variable de docentes, Metros representa el área en metros y Admin representa la variable de administración. Siendo el IRI una representación de la actividad de investigación de las instituciones de educación superior, es de esperarse que el número de docentes en las mismas afecte de manera directa a este índice, pues en general la investigación en una universidad es realizada principalmente por ellos. Además, se sabe que el tamaño de la planta física universitaria, como indicador de capacidad, pudiera 
92 Andrea González Villar, Josefa Ramoni Perazzi \& Giampaolo Orlandoni Merli

Tabla 2: Resumen de las estimaciones del Modelo Final para el IRF. Fuente: elaboración propia.

\begin{tabular}{llllll}
\hline Parámetro & $\begin{array}{l}\text { Coeficiente } \\
\text { Estimado }\end{array}$ & $\begin{array}{l}\text { Desviación } \\
\text { Estándar }\end{array}$ & $z$ & $\begin{array}{l}\text { valor } \\
p\end{array}$ & $\begin{array}{l}\text { Coeficiente } \\
\text { Estandar. }\end{array}$ \\
\hline$\beta_{1}$ & 0.102 & 0.039 & 2.590 & 0.010 & 0.042 \\
$\beta_{2}$ & 0.157 & 0.060 & 2.590 & 0.010 & 0.009 \\
$\beta_{3}$ & 0.115 & 0.042 & 2.730 & 0.006 & 0.005 \\
\hline$\mu$ & 3.216 & 0.361 & 8.910 & 0.000 & \\
$\eta$ & 0.006 & 0.021 & 3.230 & 0.001 & \\
llgt $Y$ & 1.076 & 0.286 & 3.760 & 0.000 & \\
$Y$ & 0.745 & 0.054 & & & \\
\hline
\end{tabular}

contribuir en la labor de investigación, por ejemplo en el caso de laboratorios o espacios físicos dedicados a esta actividad, por lo que se pudiera presumir que los metros cuadrados de la universidad afecten al IRI. A la espera de que los recursos administrativos y financieron afecten positivamente el IRI, pues en función de estos recursos se asigna el presupuesto dirigido a investigación.

En la tabla2 2se muestra que los parámetros del modelo son altamente significativos. El parámetro de ineficiencia técnica es significativo, indicando que los efectos de ineficiencia técnica tienen impacto en el IRI. Además, el parámetro de efectos de ineficiencia técnica variante en el tiempo $\eta$, resultó ser altamente significativo, lo que indica que el nivel de ineficiencia técnica en el IRI de las universidades estatales colombianas cambia a lo largo de los años

De aquí se tiene que, el indicador en metros cuadrados de la planta física de las universidades afecta directamente la investigación; es decir, al aumentar 1\% este indicador, el IRI se incrementará $0,115 \%$. El aumento en los recursos financieros afecta de manera positiva al índice de resultados de investigación; así, al aumentar en $1 \%$ en promedio, el índice se incrementará en un $0,102 \%$, siendo este el indicador de capacidad que tiene un mayor efecto en el índice de resultado de investigación, en relación a los otros indicadores. Y el incremento en el número de docentes tiene un efecto directo sobre el índice de resultados de investigación, y al incrementar en $1 \%$ el número de docentes, el IRI crecerá en promedio en un $0,157 \%$. Al igual que en el IRF, en el índice de resultados de investigación la UNAL y la UDEA, se alejan significativamente del resto de las instituciones, por ser las instituciones con mayor capacidad (ver figura 4.2).

La tabla 3 muestra los valores de la eficiencia técnica y la clasificación en función de la regresión cuantil en el IRF e IRI de las universidades estatales colombianas para los años 2003, 2008 y 2012. En cuanto al IRF, en forma general todas las universidades mantienen o suben su posición en el ranking general, durante el periodo 2003-2012. Dejando ver el crecimiento en los niveles de eficiencia en formación. Las universidades de Tolima, Militar de Nueva Granada, Valle, entre otras, se mantienen clasificadas con el nivel alto durante todo el periodo. Por otro lado, las universidades de Antioquia, Francisco de Paula Santander-Cúcuta, Pedagógica 
Tabla 3: Clasificación jerárquica de las universidades de acuerdo a su nivel de eficiencia técnica en los índices de resultados de formación e investigación. Valores en blanco representan eficiencia baja, en gris representan eficiencia media, en azul representan eficiencia alta. Fuente: elaboración propia.

\begin{tabular}{|c|c|c|c|c|c|c|}
\hline \multirow{2}{*}{ Instituciones de educ. superior } & \multicolumn{3}{|c|}{ Eficiencia IRF } & \multicolumn{3}{|c|}{ Eficiencia IRI } \\
\hline & 2003 & 2008 & 2012 & 2003 & 2008 & 2012 \\
\hline Universidad del Tolima & 0,00460 & 0,00532 & 0,00595 & 0,026 & 0,0295 & 0,0324 \\
\hline $\begin{array}{l}\text { Universidad Militar Nueva Gra- } \\
\text { nada }\end{array}$ & 0,00409 & 0,00474 & 0,00533 & 0,033 & 0,0365 & 0,0399 \\
\hline Universidad de Pamplona & 0,00404 & 0,00469 & 0,00527 & 0,026 & 0,0293 & 0,0322 \\
\hline $\begin{array}{l}\text { Universidad Nacional Abierta a } \\
\text { Distancia }\end{array}$ & 0,00396 & 0,00460 & 0,00517 & 0,021 & 0,0239 & 0,0264 \\
\hline Universidad del Valle & 0,00388 & 0,00451 & 0,00506 & 0,044 & 0,0493 & 0,0535 \\
\hline $\begin{array}{l}\text { Universidad Nacional de Colom- } \\
\text { bia }\end{array}$ & 0,00369 & 0,00429 & 0,00483 & 0,096 & 0,1039 & 0,1104 \\
\hline Universidad de Antioquia & 0,00354 & 0,00412 & 0,00464 & 0,054 & 0,0592 & 0,0639 \\
\hline Universidad del Magdalena & 0,00319 & 0,00372 & 0,00420 & 0,031 & 0,0352 & 0,0385 \\
\hline $\begin{array}{l}\text { Universidad Distrital Francisco } \\
\text { José de Caldas }\end{array}$ & 0,00304 & 0,00356 & 0,00402 & 0,026 & 0,0293 & 0,0322 \\
\hline $\begin{array}{l}\text { Universidad Francisco de Paula } \\
\text { Santander-Cúcuta }\end{array}$ & 0,00302 & 0,00353 & 0,00399 & 0,028 & 0,0313 & 0,0344 \\
\hline $\begin{array}{l}\text { Universidad Pedagógica y Tec- } \\
\text { nológica de Colombia }\end{array}$ & 0,00292 & 0,00342 & 0,00386 & 0,025 & 0,0288 & 0,0317 \\
\hline Universidad de Caldas & 0,00279 & 0,00326 & 0,00370 & 0,037 & 0,0411 & 0,0448 \\
\hline Universidad de Nariño & 0,00276 & 0,00323 & 0,00366 & 0,029 & 0,0332 & 0,0364 \\
\hline $\begin{array}{l}\text { Universidad Pedagógica Nacio- } \\
\text { nal }\end{array}$ & 0,00266 & 0,00312 & 0,00354 & 0,038 & 0,0426 & 0,0464 \\
\hline $\begin{array}{l}\text { Universidad Industrial de San- } \\
\text { tander }\end{array}$ & 0,00260 & 0,00305 & 0,00346 & 0,031 & 0,0346 & 0,0379 \\
\hline Universidad Surcolombiana & 0,00243 & 0,00286 & 0,00324 & 0,030 & 0,0340 & 0,0372 \\
\hline $\begin{array}{l}\text { Universidad Tecnológica de Pe- } \\
\text { reira }\end{array}$ & 0,00243 & 0,00285 & 0,00324 & 0,032 & 0,0359 & 0,0393 \\
\hline Universidad del Quindío & 0,00240 & 0,00283 & 0,00321 & 0,032 & 0,0360 & 0,0394 \\
\hline Universidad de la Guajira & 0,00214 & 0,00253 & 0,00288 & 0,035 & 0,0396 & 0,0432 \\
\hline Universidad de Cartagena & 0,00211 & 0,00249 & 0,00283 & 0,028 & 0,0317 & 0,0348 \\
\hline Universidad del Atlántico & 0,00204 & 0,00241 & 0,00274 & 0,028 & 0,0317 & 0,0348 \\
\hline $\begin{array}{l}\text { Universidad Tecnológica del } \\
\text { Choco }\end{array}$ & 0,00203 & 0,00240 & 0,00274 & 0,030 & 0,0337 & 0,0369 \\
\hline Universidad Popular del Cesar & 0,00202 & 0,00239 & 0,00272 & 0,029 & 0,0321 & 0,0353 \\
\hline Universidad del Cauca & 0,00181 & 0,00215 & 0,00246 & 0,029 & 0,0330 & 0,0362 \\
\hline $\begin{array}{l}\text { Universidad Francisco de Paula } \\
\text { Santander-Ocaña }\end{array}$ & 0,00181 & 0,00215 & 0,00246 & 0,041 & 0,0459 & 0,0499 \\
\hline Universidad de Cundinamarca & 0,00175 & 0,00207 & 0,00237 & 0,026 & 0,0295 & 0,0324 \\
\hline $\begin{array}{l}\text { Universidad Colegio Mayor de } \\
\text { Cundinamarca }\end{array}$ & 0,00173 & 0,00205 & 0,00235 & 0,033 & 0,0370 & 0,0405 \\
\hline Universidad de los Llanos & 0,00168 & 0,00199 & 0,00228 & 0,035 & 0,0387 & 0,0422 \\
\hline Universidad del Pacifico & 0,00163 & 0,00193 & 0,00221 & 0,048 & 0,0529 & 0,0572 \\
\hline Universidad de la Amazonía & 0,00160 & 0,00190 & 0,00218 & 0,030 & 0,0333 & 0,0365 \\
\hline Universidad de Córdoba & 0,00157 & 0,00187 & 0,00215 & 0,028 & 0,0314 & 0,0345 \\
\hline Universidad de Sucre & 0,00142 & 0,00170 & 0,00195 & 0,037 & 0,0410 & 0,0447 \\
\hline
\end{tabular}


94 Andrea González Villar, Josefa Ramoni Perazzi \& Giampaolo Orlandoni Merli

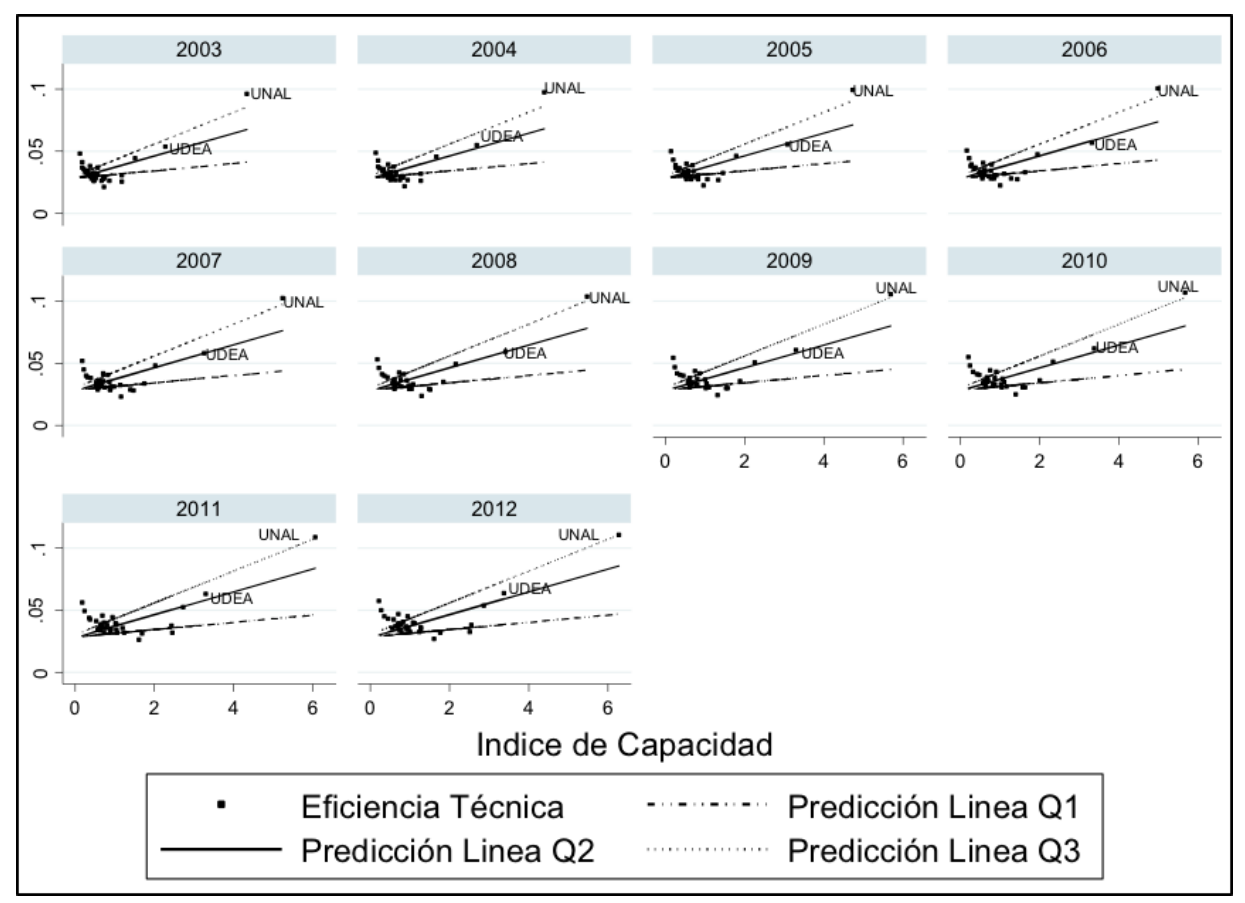

Figura 2: Disposición de las fronteras estocástica de producción del IRI a través de la regresión cuantíl, para las universidades estatales colombianas durante el período 2003-2012. Datos MEN. Cálculos propios. Fuente: elaboración propia.

y Tecnológica de Colombia, Caldas, Nariño y Pedagógica Nacional ascendieron durante el último año al nivel alto de clasificación en este índice de resultados. Y para el año 2008, algunas instituciones crecieron en formación pasando del nivel y en relación con los recursos monetarios, tal es el caso de las universidades de Cartagene, Atlántico, Tecnológica del Choco y Popular del Cesar.

Con relación a la eficiencia técnica de la actividad de investigación de las instituciones de educación superior publicas colombianas, tenemos que las universidades clasificadas con eficiencia alta se mantienen en el mismo nivel a lo largo de los años, se pueden citar las universidades Nacional de Colombia, Caldas, Pedagógica Nacional, Guajira, Francisco de Paula Santander-Ocaña, Los Llanos, Pacifico y Sucre. Es importante resaltar que no se encontraron instituciones que asciendan a este nivel. Por el contrario, se puede observar que al menos una cuarta parte de las universidades que en el año 2003 clasificaron como instituciones con eficiencia baja, para el año 2008 suben al nivel de eficiencia media, y con eso muestran los esfuerzos que se hacen en el área de investigación; en este grupo las universidades que pueden resaltarse son la Francisco de Paula Santander-Cúcuta, Popular del Cesar, Cauca, Amazonía y Córdoba. 
También se tiene un grupo más pequeño de instituciones que se clasifican a lo largo de todo el período con niveles bajos de eficiencia técnica, tal es el caso de las universidades del Tolima, Pamplona, Nacional Abierta a Distancia, Distrital Francisco José de Caldas, Pedagógica y Tecnológica de Colombia y de Cundinamarca.

Ahora veamos, en la tabla 4 , el compartamiento de las instituciones en función de las variaciones promedio interanuales de estos índices para el período en estudio. Para ello las universidades se organizan en forma jerárquica en función de su capacidad. La clasificación de las universidades estatales colombianas a través del índice de capacidad presenta cuatro grupos comparables. Un primer grupo de universidades muy grandes con un IRC mayor a 2 y un segundo de grupo de instituciones grandes con IRC entre 0.90 y 2. El tercer grupo lo conforman instituciones de capacidad media con IRC entre 0.50 y 0.90 ; dejando al resto de las universidades consideradas pequeñas con IRC menor a 0.50 .

La UNAL y la UDEA forman el grupo de las instituciones muy grandes, con variaciones promedio interanuales para el IRF que las ubica en las posiciones 27 y 26 , respectivamente, y para el IRI quedan ubicadas en las posiciones 32 y 31 , respectivamente.

En el grupo de universidades grandes, la Universidad Industrial de Santander se ubica en la posición 18 en el IRI y 17 en la posición 29 para el IRF, mostrándola como una institución que ha tenido un crecimiento medio de la eficiencia técnica. Pero destacándose en el índice de formación en relación a las instituciones que se encuentran en este mismo grupo, pues es la Universidad Pedagógica y Tecnológica de Colombia la que presenta un mejor nivel de eficiencia técnica en el IRI, inclusive comparándola a nivel general, dado que se ubica en la posición dos del ranking general para este índice.

En las universidades de capacidad media, es la Universidad de Córdoba la que muestra crecimientos altos de la eficiencia técnica en investigación y formación, pues se ubica en la segunda y octava posición, respectivamente. Mientras que la Universidad Nacional Abierta a Distancia tiene el mayor crecimiento en el nivel de eficiencia en el índice de resultado de investigación, ubicada en la primera posición en el ranking general.

Finalmente, en el grupo de universidades pequeñas la Universidad de Sucre presenta el mayor crecimiento de la eficiencia en formación, ubicada en la primera posición. Contrariamente, esta institución se ubica en los últimos lugares de la variación promedio interanual para el índice de investigación. Un comportamiento similar a esta institución lo presenta la Universidad Francisco de Paula SantanderOcaña (UFPSO) mientras que la Universidad Militar de Nueva Granada se ubica en las últimas posiciones para las variaciones interanuales del IRF e IRI. 
Tabla 4: Clasificación jerárquica de las universidades de acuerdo al índice de capacidad. Fuente: elaboración propia.

\begin{tabular}{|c|c|c|c|c|c|}
\hline \multirow{2}{*}{ Instituciones de educ. superior } & \multirow{2}{*}{ IC } & \multicolumn{4}{|c|}{ Variación promedio interanual } \\
\hline & & IRF & Posición & IRI & Posición \\
\hline & & & & & \\
\hline Universidad del Valle & 1,769 & 0,030 & 28 & 0,021 & 29 \\
\hline $\begin{array}{l}\text { Universidad Industrial de Santan- } \\
\text { der }\end{array}$ & 1,320 & 0,032 & 18 & 0,023 & 17 \\
\hline $\begin{array}{l}\text { Universidad Pedagógica y Tec- } \\
\text { nológica de Colombia }\end{array}$ & 1,113 & 0,032 & 22 & 0,025 & 2 \\
\hline $\begin{array}{l}\text { Universidad Distrital Francisco } \\
\text { José de Caldas }\end{array}$ & 0,917 & 0,031 & 24 & 0,025 & 4 \\
\hline Universidad de Cartagena & 0,884 & 0,033 & 13 & 0,024 & 9 \\
\hline Universidad del Cauca & 0,813 & 0,034 & 9 & 0,024 & 12 \\
\hline Universidad del Atlántico & 0,809 & 0,034 & 12 & 0,024 & 10 \\
\hline Universidad de Córdoba & 0,743 & 0,035 & 2 & 0,024 & 8 \\
\hline Universidad del Tolima & 0,706 & 0,029 & 32 & 0,025 & 6 \\
\hline $\begin{array}{l}\text { Universidad Nacional Abierta a } \\
\text { Distancia }\end{array}$ & 0,695 & 0,030 & 29 & 0,026 & 1 \\
\hline Universidad de Caldas & 0,679 & 0,032 & 21 & 0,022 & 26 \\
\hline $\begin{array}{l}\text { Universidad Tecnológica de Perei- } \\
\text { ra }\end{array}$ & 0,603 & 0,033 & 16 & 0,023 & 19 \\
\hline Universidad de Pamplona & 0,595 & 0,030 & 30 & 0,025 & 3 \\
\hline Universidad Pedagógica Nacional & 0,473 & 0,032 & 19 & 0,022 & 27 \\
\hline Universidad del Quindío & 0,470 & 0,033 & 15 & 0,023 & 20 \\
\hline Universidad de Nariño & 0,465 & 0,032 & 20 & 0,024 & 13 \\
\hline Universidad Popular del Cesar & 0,430 & 0,034 & 10 & 0,024 & 11 \\
\hline Universidad de Cundinamarca & 0,414 & 0,034 & 7 & 0,025 & 5 \\
\hline $\begin{array}{l}\text { Universidad Tecnológica del Cho- } \\
\text { co }\end{array}$ & 0,386 & 0,034 & 11 & 0,024 & 15 \\
\hline Universidad Surcolombiana & 0,386 & 0,033 & 17 & 0,024 & 16 \\
\hline Universidad del Magdalena & 0,384 & 0,031 & 25 & 0,023 & 18 \\
\hline $\begin{array}{l}\text { Universidad Militar Nueva Grana- } \\
\text { da }\end{array}$ & 0,382 & 0,030 & 31 & 0,023 & 21 \\
\hline Universidad de la Amazonía & 0,375 & 0,035 & 3 & 0,024 & 14 \\
\hline $\begin{array}{l}\text { Universidad Francisco de Paula } \\
\text { Santander-Cúcuta }\end{array}$ & 0,334 & 0,031 & 23 & 0,024 & 7 \\
\hline $\begin{array}{l}\text { Universidad Colegio Mayor de } \\
\text { Cundinamarca }\end{array}$ & 0,305 & 0,035 & 6 & 0,023 & 22 \\
\hline Universidad de los Llanos & 0,286 & 0,035 & 5 & 0,023 & 23 \\
\hline Universidad de la Guajira & 0,192 & 0,033 & 14 & 0,022 & 24 \\
\hline Universidad de Sucre & 0,161 & 0,036 & 1 & 0,022 & 25 \\
\hline $\begin{array}{l}\text { Universidad Francisco de Paula } \\
\text { Santander-Ocaña }\end{array}$ & 0,120 & 0,034 & 8 & 0,021 & 28 \\
\hline Universidad del Pacifico & 0,066 & 0,035 & 4 & 0,020 & 30 \\
\hline
\end{tabular}




\section{Conclusiones}

En esta investigación se ha tratado el tema de la evolución de la eficiencia técnica de las universidades estatales colombianas para los índices de formación e investigación en función de su capacidad, estos resultados sugieren que:

Las universidades estatales colombianas han estado experimentando crecimientos continuos en formación e investigación, desde el año 2003. Esto se ve reflejado en los cambios de los niveles de eficiencia técnica para estos procesos a lo largo del período 2003-2012. En este sentido, el modelo de fronteras estocásticas de producción con efectos de ineficiencia variante en el tiempo presenta una gran ventaja sobre los análisis de datos de corte transversal, que no permite ver la evolución de los niveles de eficiencia en el tiempo.

Los índices de formación e investigación están determinados positivamente por los recursos financieros, la planta física y los docentes, estos últimos con mayor influencia sobre estos procesos académicos. Precisamente, cada aumento en la capacidad de una universidad produce un incremento en los procesos de formación e investigación.

Algunas universidades presentan niveles altos de eficiencia, pero con tasa muy bajas de variación interanual de esos niveles de eficiencia, como es el caso de La Universidad del Pacifico que no ofrece programas de posgrado, y que en general presenta niveles bajos de eficiencia en formación para todos los años, pero es la universidad con una de las mayores tasas de variación promedio interanual de sus niveles de eficiencia, siendo esto reflejo del esfuerzo que hace esta institución por mejorar sus procesos de formación. Por otro lado, obtiene niveles altos de eficiencia, mostrándose como una institución orientada en los últimos años al fortalecimiento de la investigación.

El que los niveles de eficiencia formación e investigación de las universidades más grandes en capacidad (Nacional de Colombia y de Antioquia) queden ubicadas en los cuartiles más bajos, es señal de menores impactos marginales de los recursos sobre la eficiencia en estos índices, lo cual se traduce en la dificultad que presentan estas instituciones para aumentar su eficiencia con cada incremento en sus recursos. Las universidades del Valle, Industrial de Santander y Distrital Francisco José de Caldas son instituciones grandes en relación a su capacidad, y mantienen su nivel de clasificación en el índice de formación durante todo el periodo. La Universidad Pedagógica y Tecnológica de Colombia alcanza el nivel alto en el año 2012. Se destaca la Universidad Industrial de Santander como la más eficiente en el índice de formación de este grupo, en términos de sus recursos disponibles, a lo largo del periodo bajo estudio.

Entre algunas de las universidades clasificadas como de capacidad media, se encuentra la Universidad de Pamplona, con nivel alto en el índice de formación, manteniéndose así desde el 2003 al 2012. No obstante, las universidades con capacidad media, reflejan niveles bajos en el índice de investigación; sin embargo es la universidad de Caldas la más eficiente en investigación en este grupo. Es impor- 
tante, destacar la Universidad de Córdoba que con niveles bajos en los indicadores y eficiencia en investigación, es la institución de este grupo con mayor tasa de variación interanual de los niveles de eficiencia, exponiendo el afán en mejorar sus procesos de investigación.

El último grupo de universidades en relación a su tamaño, están las instituciones de capacidad baja, las cuales en general muestran a través de los años niveles bajos en formación e investigación. En este grupo sobresale la Universidad Pedagógica Nacional como la de mayor eficiencia en el índice de investigación, y la Universidad Militar de Nueva granada muy eficiente en formación. La posición de estas universidades en la clasificación general se mantiene en todos los años.

El hecho de evaluar las universidades estatales colombianas continuamente produce un impacto en la actitud que se tiene frente al mejoramiento de sus procesos misionales. Pero si bien es cierto que el sistema de indicadores de gestión es un mecanismo que efectivamente incentiva al mejoramiento de las universidades, también es fundamental la calidad de información que se tenga en estos indicadores, y con estos se sugiere hacer énfasis en la revisión a los datos suministrados por las distintas universidades.

Recibido: 19 de mayo de 2016 Aceptado: 29 de septiembre de 2016

\section{Referencias}

Battese, G. \& Coelli, J. (1992), 'Frontier Production Function, Technical Efficience and Panel Data: with application to paddy farmers in India', The Journal of Productivity Analysis 3, 149-169.

Battese, G. \& Coelli, J. (1995), 'A Model for Technical Effects in a Stochastic Frontier Production Function for Panel Data', Empirical Economics 20, 325332 .

Battese, G. \& Corra, G. (1977), 'Estimation of a Production Function Model: With Application to the Pastoral Zone of Eastern Australia', Australian Journal of Agricultural Economics 21(3), 169-179.

Castilla, D. \& García, J. (2009), 'El análisis de fronteras estocásticas: orígenes y desarrollo?. V Congreso internacional de historia de la estadística y de la probabilidad', AHEPE. Universidad de Santiago de Compostela. España. .

Cervera, M., Oviedo, G. \& Pineda, A. (2013), 'Revisión bibliográfica de la aplicación de la metodología DEA en el ámbito educativo colombiano', Civilizar 13(25), 133-156.

Coelli, T. (1996), A guide to frontier version 4.1: A computer program of stochastic frontier production and cost function estimation., CEPA Working Papers 7, Department of Econometrics. University of New Englant. Australia. 
Gómez, J. (2006), La evaluación de la eficiencia en las universidades públicas espanolas, in 'X Jornadas de la asociación económica de la educación', pp. 411434.

Melo, L., Ramos, J. \& Hernández, P. (2014), 'La educación superior en Colombia: situación actual y análisis de eficiencia', Borradores de EconomBorradores de Economíaia 808, 1-50.

Montoya, J., Torres, J. \& D'Amico, R. (2002), Fronteras estocásticas, eficiencia técnica y escalas de operación en pesca demersal (pesquería de merluza común de chile), in 'Encuentro de Economía', Chile.

Ramos, J., Moreno, J., Almanza, C., Picón, C. \& Rodríguez, G. (2015), Universidades públicas en Colombia. Una perspectiva de la eficiencia productiva y la capacidad científica y tecnológica., Editorial Universidad Del Norte, Colombia.

Rencher, A. (2002), Methods of Multivariate Analysis., Wiley-Interscience, Canada. 
